\title{
LA LINGUISTICA BALCANICA IN ITALIA: ORIGINI, EVOLUZIONE E LINEE TEORICHE ${ }^{1}$
}

1. Qualsiasi analisi relativa a temi di Linguistica Balcanica (LB, d'ora in poi) in Italia risulterebbe incompleta se non si considerassero i legami che tale settore di ricerca ha avuto (ed ha ancora) con i paralleli sviluppi della ricerca balcanologica di ambiente europeo.

In altre parole: è impossibile identificare una LB “italiana” che possa essere considerata indipendente rispetto alle linee di ricerca sviluppatesi in numerosi Centri di ricerca fuori d'Italia, in Europa, soprattutto, da parte di ricercatori che si sono occupati della situazione storico-culturale e linguistica del Sud-Est europeo, ovvero dell'area di cui i Balcani sono parte integrante.

2. Per cominciare io credo che occorra considerare la ricerca italiana nel campo della Balcanologia entro parametri di riferimento che, per la loro particolare natura, devono essere etichettati come pienamente "europei" o, per essere più precisi, "mitteleuropei".

3. Come disciplina, la LB è stata formalizzata e codificata ad un livello accademico in tempi relativamente recenti. Come è noto, le sue radici sono da rintracciarsi nell'ambiente scientifico proprio della seconda metà del secolo XIX, quando, nei maggiori centri di ricerca linguistica dell'Europa tedescofona, studiosi del calibro di J. Kopitar, A. Schleicher, Fr. Miklosich, Th. Kapidan e H. Schuchardt cominciarono ad occuparsi in modo sistematico dei problemi del plurilinguismo sud-est europeo.

Non è casuale, del resto, che alcuni dei ricercatori premenzionati furono tra $i$ fondatori del primo Centro di ricerca che si occupò di problemi linguistici dell'area balcanica, considerati in prospettiva interdisciplinare: storica, etnografica, demologica. Il riferimento è alla Balkankommission viennese, che divenne celebre soprattutto grazie all'attività di Fr. Miklosich e di Th. Kapidan.

Non è una coincidenza il fatto che la Balkankommission viennese si sviluppò in stretta connessione con gli ultimi bagliori dell'espansionismo asburgico: quando, all'inizio della seconda metà del secolo scorso, mentre l'impero ottomano cominciava a declinare e a segnare definitivamente $i$ destini dell'Europa sud-orientale, i funziona-

$1 \quad$ Il presente lavoro è la rielaborazione, in molti luoghi radicale e con opportuni aggiornamenti bibliografici, di un testo di una conferenza, originariamente in lingua inglese, preparato per un Seminario di Balcanologia tenutosi a Mosca, presso l'Istituto di Slavistica e Balcanologia della GUM, nell'ottobre del 1990. 
ri dell'Austria Felix pensavano di poter conquistare ed assimilare i territori balcanici sottoposti al giogo ottomano.

Allo stesso modo, non è un caso che il primo progetto della Balkankommission viennese fu la definizione di un rigoroso programma di "politica linguistica" che si pensava di poter applicare ed estendere alle genti del Sud-Est europeo, il cui territorio fu considerato come l'area per eccellenza caratterizata da lingue e culture diverse.

Il programma era, di fatto, straordinariamente avanzato e si fondava su un reale, effettivo rispetto dell'identità etnicolinguistica, religiosa e culturale delle genti balcaniche. Inoltre, il suo primo e fondamentale scopo fu la descrizione della situazione linguistica dell'area balcanica secondo i metodi d'indagine propri della ricerca linguistica e dialettologica del tempo.

4. La LB nacque, di fatto, in ambiente tedescofono: per essere più precisi a Vienna, Lipsia e Berlino. Sulle prime la LB si sviluppò in modo assai differenziato, grazie a ricerche relative al settore morfosintattico (riduzione dei sistemi flessivi delle lingue balcaniche; sviluppo dell'articolo determinativo postposto; riduzione dell'infinito; formazione del futuro analitico). Oltre a ciò, settori di ricerca particolarmente seguiti furono i problemi relativi al lessico: definizione del lessico "balcanico" ereditario; analisi dell'influsso greco-bizantino, latino-romanzo e turco sui sistemi lessicali delle lingue balcaniche.

5. Fu soltanto durante la seconda decade del nostro secolo che apparvero le prime sintesi relative alla situazione linguistica dell'area balcanica. Mi riferisco, ovviamente, ai lavori di A. Seliščev, uno slavista bulgaro, ma, soprattutto, alla prima ampia monografia dedicata ai problemi linguistici dei Balcani, visti all'interno di un quadro di riferimento socio-culturale: il lavoro di Kr. Sandfeld, Linguistique balkanique, problèmes et résultats, apparso dapprima in edizione danese, a Kopenhagen, nel 1926; quindi fu pubblicato, in edizione francese, a Parigi, nel 1930.

I lavori di A. Seliščev e di Kr. Sandfeld contribuirono a diffondere nell' ambiente scientifico internazionale $i$ risultati delle ricerche scientifiche di ambito balcanologico condotte durante il secolo XIX e nel primo ventennio del nostro secolo. Grazie al lavoro di A. Seliščev e di Kr. Sandfeld, non possiamo dimenticare che l'area balcani$\mathrm{ca}$ - in cui si erano incontrate e fuse le lingue slave meridionali, il greco, il rumeno e il dalmatico, l'albanese, il veneziano, il turco, lo jiddish, l'armeno, i dialetti zigàni parve essere il terreno di ricerca più conveniente per studiare i meccanismi del contatto linguistico tra sistemi diversi.

Non è una coincidenza poi che, tra il 1926 e il 1928, i ricercatori della Scuola linguistica di Praga considerarono il "modello balcanico" come l'esempio più chiaro di area linguistica ove poter sperimentare le teorie relative al contatto linguistico: a loro si deve, tra l'altro, la prima definizione del concetto di Sprachbund ("lega linguistica"). 
6. Come disciplina autonomamente fondata, la LB sorse così dall'intersezione tra problemi storico-linguistici, evidenziati già dal dibattito ottocentesco, e da alcuni elementi, di diversa natura, orientati e basati su problemi teorico-generali.

Questi ultimi furono, di fatto, l'esito del dibattito scientifico che, negli anni Venti, interessò la Scuola di Praga. A livello internazionale ebbero grande eco le "tesi" del primo Congresso dei Linguisti, tenutosi a Praga nel 1928: la LB, considerata come $l a$ linguistica delle "lingue in contatto", fu utilizzata come modello teorico e come punto di riferimento principale da parte dei ricercatori che cominciavano ad occuparsi, pur su diversi terreni d'analisi, di tematiche relative al plurilinguismo.

Tale duplice interesse, storico-linguistico, da un lato, linguistico-generale, dall'altro è, oggi, la caratteristica saliente della ricerca balcanologica: ciò è testimoniato dalle linee di ricerca in generale $e$, in particolare, ciò vale per la LB italiana, caratterizzata per altro da alcune peculiarità che esporrò nel paragrafo seguente.

7. In Italia, a livello accademico, la LB non ha mai avuto, fino ad oggi (1991), un riconoscimento ufficiale: non ci sono mai stati, né ci sono, Centri di ricerca completamente dedicati agli studi sud-est europei, paragonabili agli Istituti di Balcanologia o ai Centri di ricerca presenti in quasi tutte le università dell'Europa orientale: da Mosca a San Pietroburgo; da Bucarest a Sofia; da Belgrado a Sarajevo; ma anche in Grecia,

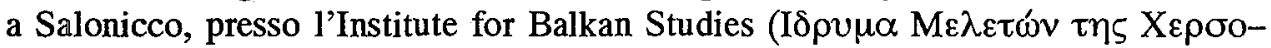

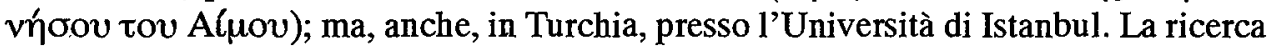
balcanologia è comunque, ben presente anche in alcuni Centri di studio dell'Europa occidentale, soprattutto in Germania: a Berlino, alla Freie Universität e alla Humboldt Universität; a Monaco, presso l'Ost-Europa Institut.

In Italia la LB è stata sempre considerata una disciplina extramoenia, una sorta di "condominio", in cui ricercatori con diversi percorsi formativi e diversi orientamenti teorici hanno lavorato e tuttora lavorano. La LB è stata soprattutto "promossa", e ancora lo è, sia da studiosi di linguistica storica e sia da classicisti. Gli studiosi di linguistica storica, ossia i comparatisti, gli indeuropeisti, interessati all' antica vicenda storica dei Balcani, considerati come "crocevia" dell'ambiente indeuropeo. I classicisti, a loro volta distinguibili in: i] studiosi che si sono occupati, e si occupano, delle fasi seriori delle lingue classiche: latinisti, interessati ai temi dell'evoluzione del latino nella sua fase tarda, preromanza; ii] studiosi di lingua greca bizantina e medievale; iii] romanisti (interessati allo studio dei rapporti tra la cosiddetta "Romania Balcanica" $e$ l'Occidente romanzo, con particolare riferimento per il processo di formazione del latino balcanico e per l'origine del rumeno e dei suoi dialetti; iv] studiosi di albanologia (interessati all'origine antica dell'albanese, ai suoi rapporti con il protorumeno e studiosi del ruolo linguistico dell'ambiente albanese nella più recente storia dei Balcani; v] slavisti (con particolare riferimento per ricercatori che si sono occupati del processo di formazione dell'ambiente bizantino e medievale e dell'ambiente romanzo-orientale); infine: vi] studiosi che si occupano di definire modelli di analisi linguistica in chiave tipologica e di tipologia comparata. 
8. Di seguito offrirò un quadro dei contributi offerti da studiosi italiani nel campo della LB. Il mio lavoro è soprattutto focalizzato sui principali temi trattati dagli studiosi italiani: esporrò i loro orientamenti teorici e i risultati raggiunti. Seguirò, nella mia esposizione, un criterio "tematico" piuttosto che un criterio cronologico.

8.1. Il primo, importante tema trattato dagli studiosi italiani, a partire dagli anni Trenta, fu la definizione della situazione linguistica dell'area balcanica prima che intervenissero i processi di grecizzazione e di latinizzazione della penisola.

In altre parole, gli studiosi italiani - da F. Ribezzo ${ }^{2}$ ad O. Parlangeli ${ }^{3}$, da V. Pisa$\mathrm{ni}^{4}$ a C. De Simone $\mathrm{s}^{5}$ - si sono occupati delle cosiddette "lingue di sostrato balcanico", ossia dell'Illirico, del Tracio, del Daco-Misio. Essi sottolinearono i reciproci contatti tra le varie lingue, $i$ loro rapporti con le altre lingue indeuropee $e$, infine, quando possibile, studiarono la loro evoluzione fino alla più recente facies delle lingue balcaniche, con particolare attenzione per i dialetti rumeni e albanesi.

In questo particolare settore vanno menzionati soprattutto alcuni studiosi: in primo luogo, di nuovo, V. Pisani, per lunghi anni attivo nelle università milanesi, i cui importanti contributi sulla preistoria dell'ambiente illirico e della sua evoluzione fino al moderno quadro linguistico albanese sono ancora di grande importanza. Vanno poi ricordati O. Parlangeli ${ }^{6}$, allievo di V. Pisani, e C. De Simone, i cui studi sulla posizione del messapico, considerato come una "derivazione" dell'Illirico in ambiente pugliese, sono degni di particolare attenzione.

La medesima linea teorica è stata poi, in anni recenti, ripresa da alcuni studiosi che hanno seguito l'impianto teorico di O. Parlangeli e di C. De Simone: in particolare, vanno menzionati i risultati delle ricerche di C. Santoro?

8.2. Il secondo orientamento della ricerca italiana nel campo della LB si è accentrato sullo studio delle componenti proto-balcaniche nella formazione degli ambienti linguistico-culturali greco e italico (pre-latino).

La scuola italiana, a questo proposito, ha prodotto alcuni studi originali il cui valore va ben al di là delle tematiche relative ai Balcani e includono anche alcuni temi connessi con il processo di formazione di tutto l'ambiente linguistico europeo-occidentale. Di nuovo, accanto a G. Devoto e a M. Laviosa Zambotti, va ricordato il contributo di V. Pisani. A loro dobbiamo la precisa definizione della funzione della componente "balcanica" osco-umbra nella formazione della componente italica e,

2 Cf. i suoi importanti, numerosi contributi apparsi in "Rivista Indo-greco-italica di filologia, lingua, antichità" (Napoli (1917-1937).

3 Cf. O. Parlangeli, La penisola balcanica e l'Italia, in Atti del III Congresso di Linguisti, Milano, 1961, pp. 12.37.

4 Tra le opere di V. Pisani dedicate al sostrato balcanico, cf. quelle citate nella nota 7.

5 Cf. C. De Simone, Die messapische Inschriften, Wiesbaden, 1964.

6 Cf. O. Parlangeli, Studi messapici, iscrizioni, lessico, glosse e indici, Milano, 1960.

7 Cf. C. Santoro, Nuovi studi messapici, voll. 3, Galatina, 1982-1984. 
inoltre, la determinazione della componente "balcanica-eolica" nel processo di formazione dell'ambiente greco pre-classico ${ }^{8}$.

Secondo questi studiosi, è assai probabile che tra il I e il II millennio a.C. si fosse formato un antichissimo Sprachbund comprendente le predette componenti: tracce della sua presenza sono visibili nella fase tarda delle lingue nelle due tradizioni linguistiche, sono evidenti nel latino volgare e nel greco volgare: come ho cercato di dimostrare in un mio lavoro, Riflessi degli antichi contatti eolico osco-umbri nel neogreco e nei dialetti italomeridionali (Atti dell'Accademia di Scienze Lettere e Arti di Palermo, Serie IV, vol. XXXV - 1977), in cui ho sviluppato alcune posizioni teoriche proposte da V. Pisani e da G. Devoto tra gli anni Cinquanta e gli anni Sessanta.

8.3. Il terzo, importante campo di ricerca sviluppato dai balcanologi italiani si riferisce ai rapporti interlinguistici tra la cosiddetta "Balcania graeco-romana" e i lembi inferiori della penisola italiana (Calabria meridionale, Puglia meridionale, Sicilia). Mi riferisco ovviamente ad un settore di ricerca che ha prodotto importanti risultati e che può essere suddiviso, per ragioni puramente espositive, in tre principali sotto-settori:

i] lo studio delle relazioni tra latino-balcanico (soprattutto nell' area danubiana, là dove si formarono i dialetti rumeni) e i moderni dialetti italo-romanzi della Calabria, del Salento, della Sicilia ${ }^{9}$;

ii] lo studio delle relazioni tra ambiente dalmatico ed ambiente italo-romanzo; con particolare attenzione per il contatto tra le due opposte sponde dell'Adriatico (un mare che fu sempre facile da attraversare e che facilitò, piuttosto che ostacolare, $i$ rapporti tra Italia e i Balcani).

In questo particolare settore di ricerca occorre ovviamente ricordare M. Bartoli, il cui lavoro è, ancor oggi, assolutamente fondamentale. Nei suoi studi sul dalmatico, pubblicato a Vienna nel 1906, egli suppose l'esistenza di una "catena" apennino-dalmatica e, soprattutto, egli collegò al dalmatico i dialetti italo-romazi parlati lungo la costa adriatica ${ }^{10}$. La teoria bartoliana è stata recentemente riconsiderata da alcuni

8 Su questo tema, cf; G. Devoto, Italo-greco ed italo-celtico, Archivio Glottologico Italiano, XXII-XXII (1929), pp. 200-240; Id., Gli antichi italici, Firenze, 1931; Id., Problemi e orientamenti di grammatica storica e di storia delle lingue classiche, Milano, 1951. Tra i lavori di V. Pisani intorno a questi temi, vanno menzionati, almeno, i seguenti: V. Pisani, Le lingue dell'Italia antica oltre il latino, Torino, 1953; Id. Über eine pälignische Inschrift (Co. 208 bis. Pl. 246 d) und die Herkanft des Oskisch-Umbrischen, "Rheinisches Museum", XCV (1952), pp. 1-22 (anche in Saggi di Linguistica Storica, Torino, 1959, pp. 137-159); Id., Le lingue indeuropee in Grecia e in Italia, "Rendiconti dell'Istituto Lombardo" 89 (1956), pp. 93-112; Id., Zum Sprachgeschichte des alten Italiens, "Rheinisches Museum", XCVII (1954), pp. 47-68. Infine, va ricordato l'importante lavoro di M. Laviosa Zambotti, I Balcani el'Italia nella Preistoria. Premessa alla comprensione del mondo classico, Como, 1954.

9 Cf; G. Alessio, Concordanze lessicali tra i dialetti rumeni e quelli calabresi, "Annali della Facoltà di Lettere e Filosofia dell'Università di Bari’' I(1954), pp. 3-53; E. Banfi, A ree latinizzate nei Balcani e una terza area latino-balcanica (area della via Egnazia), "Rendiconti dell'Istituto Lombardo Accademia di Scienze e Lettere LII (1972). 
dialettologi italiani (A. Melillo e M. Giammarco) ${ }^{11}$. Questi studiosi hanno sottolineato le straordinarie affinità, a livello fonetico, tra dialetti italo-adriatici e alcuni elementi dalmatici tramandatici dal Bartoli e, soprattutto, da alcune testimonianze presenti nelle "lettere Zaratine", le più antiche fonti scritte relative a questa lingua.

Accanto ai lavori del Bartoli occorre ricordare gli studi di C. Merlo ${ }^{12}$, dialettologo pisano, che prese in considerazione i rapporti tra dialetti dalmatici e dialetti italosettentrionali in una serie di importanti saggi (talvolta assai polemici nei confronti del Bartoli). Secondo il Merlo, il dalmatico altro non è se non un anello di una catena romanza-settentrionale che lega i parlari retoromanzi, ladini e friulani con i parlari romanzi danubiani, ossia con l'area linguistica da cui prese origine il rumeno.

iii] infine, l'ultimo settore di ricerca ampiamente percorso da studiosi italiani si riferisce alle relazioni tra la componente latino-romanza e greco-bizantina in area balcanica.

Tale argomento è stato trattato soprattutto alla luce del processo di cristianizzazione del Sud-Est europeo. Ciò prevede la considerazione di una fase antica (secc. II-IV d.C.) durante la quale i Balcani erano ancora parte delle dinamiche di irradiazione romana. Quindi, un altro elemento preso in considerazione, è stato il secondo momento del processo di cristianizzazione dei Balcani (secc. VII-IX), avvenuto in un contesto politico e culturale rinnovato: i Balcani erano completamente indipendenti da Roma e già ampiamente dominati dalle componenti avaro-slave. Il messaggio cristiano vi si diffuse muovendo sia dai poli della cristianità bavarese, romano-germanica (Salisburgo e Krems, soprattutto), sia dai poli della cristianità bizantina (Bisanzio; i centri monastici bizantino-slavi).

Dal punto di vista linguistico, sulla base di lavori di A. Zamboni, di G.B. Pellegrini e miei ${ }^{13}$, quest'ultima fase della cristianizzazione sud-est europea ebbe una fun-

10 Cf. Matteo Bartoli, Das Dalmatische, Altromanische Sprachreste im Veglia bis Ragusa und ihre Stellung in der Apennino-balkanischen Romania, Wien, 1906.

11 Sull'argomento, in generale, con ampi riferimenti bibliografici, cf. C. Tagliavini, Le origini delle lingue neolatine, Bologna, 1982, pp. 374-377.

12 cf. C. Merlo, Studi glottologici, Pisa 1934.

13 Su questo argomento, cf. G.B. Pellegrini, Introduzione alla lingua albanese, Padova, 1977; A. Zamboni, Note linguistiche dalmatiche, "Atti della tomata di studio della Società Dalmata di Storia Patria”, Venezia, 1976, pp. 9-65; E. Banfi, Aree latinizzate nei Balcani e una terza area latino-balcanica (area della via Egnazia), "Rendiconti dell'Istituto Lombardo - Accademia di Scienze e Lettere - Classe di Lettere”, 106 (1972), pp. 185-243; E. Banfi, Problemi di fonetica delle aree latino-balcaniche, in H.M. Ölberg (ed.), Akten des internationalen albanologischen Kolloquiums zum Gedächtnis an $N$. Jokl, Innsbrucker Beiträge zur Kulturwissenschaft, Sonderheft 41 (1977), pp. 269-285; E. Banfi, Note sull'elemento lessicale neogreco di origine latina alla luce della romanizzazione interadriatica, “Abruzzo" 19,3 (1981), pp. 79-102; E. Banfi, Linguistica balcanica, Bologna, 1985; Id., Cristianizzazione nei Balcani e formazione della lega linguistica balcanica, "Zeitschrift für Balkanologie" 23/1 (1987), pp. 2-18.; E. Banfi, Intorno al concetto di confine linguistico latino-greco nei Balcani, "Die Slawischen Sprachen" 11 (1987), pp. 5-24; E. Banfi, Per la storia del confine linguistico greco-latino nei Balcani, "Zeitschrift für Balkanologie" 24/1 (1988), pp. 144-131; E. Banfi, Storia linguistica del Sud-Est europeo, Crisi della Romània 
zione fondamentale nel processo di stabilizzazione della situazione linguistica dei Balcani in età medievale e nella formazione di una "Balcanica romano-germanica" opposta ad una "Balcanica greco-ortodossa". La prima influenzò le componenti slovena, croata, dalmatica, albanese settentrionale; la seconda le componenti serba, macedone, bulgara, albanese meridionale, rumena.

8.4. Inoltre, gli studiosi italiani hanno esaminato anche i rapporti tra l'ambiente balcanico esposto all'influsso greco-balcanico e le regioni balcaniche caratterizzate dalla presenza di componenti slavo-meridionali e valacche: in questa prospettiva, attenta ai portati delle ricerche storiche e demologiche, occorre tener presenti alcune teorie di C. Tagliavini (i cui contributi sull'elemento balcanico nell'ungherese sono assai importanti) ${ }^{14}$ e alcune ricerche di G.B. Pellegrini e mie personali. Nel mio saggio La sostanziale balcanizzazione del territorio romeico e della lingua neogreca ("Memorie dell'Istituto Lombardo - Accademia di Scienze e Lettere, Classe di Lettere - Scienze Sociali e Storiche", XXXVII/5 . 1982, pp. 285-324), ho suggerito di analizzare l'ambiente greco (bizantino, post-bizantino, medievale e moderno) all'interno della realtà balcanica in modo da trovare una chiave interpretativa per la comprensione della situazione linguistica e culturale della Grecia medievale e moderna. Sono infatti convinto che, anche sul piano linguistico, il mondo neogreco non possa essere compreso se non a patto che esso venga correttamente inserito entro le dinamiche socio-culturali e linguistiche dell'area balcanica.

In area balcanica, l'amministrazione bizantina, erede della politica romano-imperiale, creò una vera e propria "unità" culturale. Tale "unità" è riflessa nell'organizzazione dell'ambiente balcanico medievale (origine ed evoluzione delle genti slave meridionali, dell'ambiente rumeno, dell'ambiente albanese). Quindi, dopo che la funzione del mondo bizantino divenne irrilevante all'interno dei Balcani, un simile ruolo fu assunto dalla componente turca, che giocò una funzione privilegiata, di polarizzazione per gran parte dell'area balcanica tra i secc. XV e XIX (ma, in alcuni casi, in particolare nella Grecia del Nord, fino alla metà del primo decennio del nostro secolo). Sono convinto sempre più che la componente turca, caratterizzata da un'organizzazione solida e sovrannazionale, già propria dell'impero bizantino, contribuì a creare una forte rete di legami linguistici, culturali, politici.

Dopo la caduta dell'impero ottomano e dopo che le genti balcaniche ripresero la loro specifica identità nazionale, in un'atmosfera di forte ricerca della propria tradizione storico-culturale, alcuni studiosi greci, slavi, rumeni, albanesi tentarono di limitare il ruolo della componente turca nella formazione delle loro specifiche realtà nazionali. Tuttavia, io credo che sia compito dei linguisti, sulla base di dati concreti, il ricondurre la ricerca di ambito balcanico entro parametri oggettivi, non viziati da atteggiamenti di sterile nazionalismo: una storia comparata delle lingue balcaniche

balcanica tra alto e basso medioevo, Franco Angeli, Milano, 1991.

Cf. C. Tagliavini, Le origini, cit., pp. 324-325; G.B. Pellegrini, Introduzione, cit., pp. 38-42. 
non può se non essere basata su questa "rivoluzione copernicana", per il cui avvento, io credo, i tempi siano maturi.

8.5. Un altro campo d'indagine privilegiato dagli studiosi italiani di cose balcaniche si riferisce al settore dell'albanologia. Sull'evoluzione dell'albanese dobbiamo a V. Pisani pagine ancor oggi fondamentali: Pisani, sulla base di considerazioni prevalentemente lessicologiche, sostenne l'ipotesi dell'autoctonia delle genti albanesi, eredi dell'antica tradizione illirica ${ }^{15}$.

Nel settore albanologico, particolare menzione deve essere data ai lavori di C. Tagliavini e di G.B. Pellegrini, maestri insigni dell'ateneo patavino: in particolare vanno ricordati i loro saggi sulla stratificazione del lessico albanese, la cui straordinaria complessità prevede elementi greci (pre)classici, elementi latini (di epoche diverse), elementi greco-bizantini, slavi, turchi ${ }^{16}$.

Importanti, ancora, i lavori di G. Valentini e di P. Di Giovine: al primo si deve la pubblicazione dei documenti relativi all'ambiente albanese derivati dagli archivi medievali (una vera miniera di dati, di interesse sia storico che linguistico); al secondo si devono importanti contributi alla definizione della fonetica storica dell'albanese ${ }^{17}$.

8.6. Negli anni Ottanta gli studi di LB in Italia si sono soprattutto aperti a tematiche linguistico-generali: il dibattito sui cosiddetti "balcanismi", sulla loro natura storico-linguistica, ha portato alcuni studiosi ad inserire tali problemi entro i principi della Tipologia Linguistica. P. Ramat ${ }^{18}$ ha cercato di fornire un'interessante definizione tipologica della situazione storico-linguistica dei Balcani. Di orientamento tipologico, con una costante attenzione, tuttavia, per i dati storici, sono anche alcuni miei recenti lavori ${ }^{19}$, lo stimolante saggio di G. Raffo ${ }^{20}$ e i saggi, intorno al concetto di Sprachbund, di V. Orioles ${ }^{21}$.

15 Cf. V. Pisani, L'Albanais et les autres langues indo-européennes in "Saggi di Linguistica Storica”, Torino, 1959, pp. 96-114; Id., Lexikalische Beziehungen des Albanesischen zu den anderen indogermanischen Sprachen, in "Saggi di Linguistica Storica", Torino, 1959, pp. 115-136.

16 Cf. C. Tagliavini, La stratificazione della lingua albanese, Bologna, 1966; G.B. Pellegrini, Introduzione, cit.

17 Cf. G. Valentini, Acta Albaniae Juridica, München, voll. 2, 1968-1973; Id., Acta Albaniae Veneta saec. XIV et XV, München, voll. 30, 1967; P. Di Giovine, Il gruppo CT latino in albanese, Roma, 1982.

18 Cf. Paolo Ramat, Il tipo linguistico balcanico, "Lingua e Stile" XXIII/2 (1988), pp. 301-311.

19 Cf. E. Banfi, Tipologia della convergenza linguistica in area balcanica, "Atti del Convegno della Società Italiana di Glottologia", Bergamo, 17-19 XII 1987, Pisa, 1988, pp. 43-91; Id. Typologies of Standardization in the Balkan languages, The case of two Balkanisisms; the loss of the infinitive and the periphrastic future, "Eu ropa Orientalis" 8 (1989), pp. 175-189; Id., The infinitive in South East European languages, in Bechert, Bernini, Buridiant (eds.), Toward a Typology of European Languages, Berlin-New York-Amsterdam, 1990, pp. 1-32.

21 Cf. V. Orioles, Saussure e i contatti fra lingue, "Incontri Linguistici" XVIII (1990-91), 165-176; Id., Alle origini delle nozioni di convergenza e lega linguistica, in "Studi Linguistici Salentini" XVII (1989/90), pp. 163-177. 
Nell'analisi dei "balcanismi", considerati come esempi di "tipi linguistici", gli studiosi italiani ne hanno studiato la distribuzione geografica, la loro penetrazione in diversi ambiti linguistici, la loro appartenenza ai livelli sia popolari che dotti/sorvegliati.

In particolare, $i$ linguisti italiani hanno cercato di determinare se $i$ "balcanismi" siano l'esito di un processo di convergenza linguistica originatosi da matrice popolare (determinata da influssi "dal basso", colleganti tra di loro le diverse lingue balcaniche) oppure se essi siano il risultato di un processo di influssi colti o semi-colti (determinati da influssi 'dall'alto', imposti sulle diverse lingue balcaniche dalla graduale evoluzione delle scriptae e da ampi fenomeni di omologazione culturale, trasmessi rispettivamente dalle componenti latino-balcanica, slava, greco-bizantina e, infine, turca.

Particolare attenzione è stata data soprattutto al ruolo "standardizzatore" imposto, in ambiente balcanico, dagli orientamenti religiosi, propri di singoli gruppi etnico-linguistici (ortodossia vs. cattolicesimo/protestantesimo) e dall'adozione di specifici modelli amministrativi (bizantino vs. romano-germanico).

\section{Povzetek \\ BALKANISTIKA V ITALIJI: ZAČETKI, RAZVOJ, TEORETIČNE POSTAVKE}

Balkanistika se je rodila na germanskih univerzah, vendar ima tudi italijansko jezikoslovje z $\mathbf{M}$. Bartolijem pomembnega raziskovalca jezikovne stvarnosti na Balkanu. $Z$ jezikoslovnimi problemi balkanskih jezikov so se ukvarjali številni italijanski jezikoslovci (V. Pisani, G. Devoto, C. Tagliavini, G.B. Pellegrini, E. Banfi). Nekateri zaradi poznoantične faze grščine in latinščine, drugi zaradi črt, ki jih kažeta italska jezika oskijščina in umbrijščina in imajo nekaj skupnega z balkanskimi jeziki, večina pa zaradi izoglos med balkanskimi jeziki in južnoitalijanskimi narečji. Avtor opozarja na dve različnni fazi pokristjanjenja Balkana: prva je nekako sočasna rimskemu osvajanju Dakije, ob drugem valu (VII-IX stoletje) pa Rim ne igra prav nobene vloge in torej tudi jezikovno ne more vplivati. 